\title{
Eradication of Pseudomonas aeruginosa in cystic fibrosis patients
}

\section{To the Editors:}

We share the opinion that the optimal therapeutic strategy to eradicate Pseudomonas aeruginosa from the airways of patients with cystic fibrosis (CF) is still unclear. This situation is not likely to change by comparing small studies employing different methodologies in different settings, particularly our study [1] with that of EBER et al. [2]. Clearly, larger studies have to be undertaken to provide a better basis for a consensus in this context.

We do not share the opinion of EBER et al. [2] that "true eradication" of $P$. aeruginosa is not proven in our study. Knowing that throat/sputum cultures might be false-negative, we typed sequential $P$. aeruginosa isolates from CF patients and demonstrated that the majority of $P$. aeruginosa strains, isolated after eradication therapy and a period with negative throat/ sputum cultures, were indeed genotypically different from the initial strains. Similar results have also been obtained from other investigators [3].

Furthermore, in CF patients who were not recolonised by $P$. aeruginosa in our study, the mean follow-up period was $37 \pm 29$ months. During this period, the mean number of negative throat/sputum cultures obtained from these patients was $12 \pm 8$. This number is higher than that recommended by the UK Trust [4] for assessing P. aeruginosa eradication after early antibiotic therapy (three negative respiratory cultures within a period of 6 months). Finally, antibody values derived from a 23-yr-old radioimmunoassay, which become positive only 6-15 months after the onset of $P$. aeruginosa infection, cannot be compared with those derived from the ELISA used in our study, in which the cut-off value was differently set. Indeed, our unpublished data show that some CF patients reveal positive antibody titres at the time of first infection using this ELISA. Furthermore, we have shown that antibody titres significantly decreased after successful treatment of the first $P$. aeruginosa infection [5]. Other ELISAs show that serum antibody titres in CF patients become positive even before detection of the pathogen in throat/sputum cultures [6]. Therefore, we agree that it might be misleading to rely solely on antipseudomonal serum titres to assess bacterial eradication.

Taken together, the absence of $P$. aeruginosa from many throat/ sputum cultures during a considerably long period of investigation, the typing results of consecutive patients' isolates and negative serum antibody titres against $P$. aeruginosa convincingly show that "true eradication" has indeed occurred, at least in the majority of patients studied. The suggestion of EBER et al. [2] to use bronchoalveolar lavage fluid samples for assessing eradication will certainly not be feasible for many CF centres in the world once this therapeutic strategy is applied more frequently.

Finally, we appreciate the thoughtful comments of EBER et al. [2] and hope that this will encourage other cystic fibrosis centre directors to implement early eradication therapy for Pseudomonas aeruginosa in cystic fibrosis patients.

\section{G. Döring*, G. Taccetti" , S. Campana\#, F. Festini" ${ }^{\#}$ and M. Mascherini ${ }^{\#}$ Universitätsklinikum, Tübingen, Germany, and "Dipartimento di Pediatria, Ospedale Meyer, Firenze, Italy.}

\section{REFERENCES}

1 Taccetti G, Campana S, Festini F, Mascherini M, Döring G. Early eradication therapy against Pseudomonas aeruginosa in cystic fibrosis patients. Eur Respir J 2005; 26: 458-461.

2 Eber E, Thalhammer GH, Zach MS. Eradication of Pseudomonas aeruginosa in cystic fibrosis patients. Eur Respir J 2006; 27: 438-439.

3 Munck A, Bonacorsi S, Mariani-Kurkdjian P, et al. Genotypic characterization of Pseudomonas aeruginosa strains recovered from patients with cystic fibrosis after initial and subsequent colonization. Pediatr Pulmonol 2001; 32: 288-292.

4 Cystic Fibrosis Trust. Pseudomonas aeruginosa infection in people with cystic fibrosis. Suggestions for prevention and infection control. Bromley, Cystic Fibrosis Trust, 2001.

5 Ratjen F, Grasemann H, Döring G. The role of P. aeruginosa antibodies for defining airway infection with $P$. aeruginosa in CF patients. Pediatr Pulmonol 2004; Suppl. 27: 263A.

6 West SE, Zeng L, Lee BL, et al. Respiratory infections with Pseudomonas aeruginosa in children with cystic fibrosis: early detection by serology and assessment of risk factors. JAMA 2002; 287: 2958-2967. 